\title{
Bidirectional reflectance and VIS-NIR spectroscopy of cometary analogues under simulated space conditions
}

\author{
Bernhard Jost ${ }^{\mathrm{a}, *}$, Antoine Pommerol ${ }^{\mathrm{a}}$, Olivier Poch ${ }^{\mathrm{b}}$, Zuriñe Yoldi ${ }^{\mathrm{a}}$, Sonia \\ Fornasier $^{\mathrm{c}, \mathrm{d}}$, Pedro Henrique Hasselmann ${ }^{\mathrm{c}, \mathrm{g}}$, Clément Feller ${ }^{\mathrm{c}, \mathrm{d}}$, Nathalie \\ Carrasco $^{\mathrm{e}, \mathrm{f}}$, Cyril Szopa ${ }^{\mathrm{e}, \mathrm{f}}$, Nicolas Thomas ${ }^{\mathrm{a}}$ \\ ${ }^{a}$ Physikalisches Institut, Universität Bern, Sidlerstrasse 5, CH-3012 Bern, Switzerland \\ ${ }^{b}$ NCCR PlanetS, University of Bern, Sidlerstrasse, 5, 3012 Bern, Switzerland \\ ${ }^{c}$ LESIA, Observatoire de Paris, CNRS, UPMC Univ Paris 06, Univ. Paris-Diderot, 5 place \\ J. Janssen, 92195 Meudon Pricipal Cedex, France \\ ${ }^{d}$ Univ Paris Diderot, Sorbonne Paris Cit, 4 rue Elsa Morante, 75205 Paris Cedex 13, \\ France \\ ${ }^{e}$ UniversitV́ersailles St-Quentin ; Sorbonne Universitś, UPMC Univ. Paris 06 ; \\ CNRS/INSU, LATMOS-IPSL, 11 Boulevard dAlembert, 78280 Guyancourt, France \\ ${ }^{f}$ Institut Universitaire de France, 103 Bvd St-Michel, 75005 Paris, France \\ ${ }^{g}$ Observatorio Nacional, General Jos Cristiano 77, Sao Cristovao, Rio de Janeiro, Brazil
}

\begin{abstract}
This work is intended to be the second publication in a series of papers reporting on the spectro-photometric properties of cometary analogues measured in the laboratory. Herein, we provide in-situ hyperspectral imaging data in the $0.40-2.35 \mu \mathrm{m}$ range from three sublimation experiments under simulated space conditions in thermal vacuum from samples made of water ice, carbonaceous compounds and complex organic molecules. The dataset is complemented by measurements of the bidirectional reflectance in the visible $(750 \mathrm{~nm}) \mathrm{spec}-$ tral range before and after sublimation. A qualitative characterization of surface evolution processes is provided as well as a description of morphological changes during the simulation experiment.
\end{abstract}

The aim of these experiments is to mimic the spectrum of comet $67 \mathrm{P} / \mathrm{Churyumov}$ Gerasimenko (67P) as acquired by the Rosetta mission by applying sublimation experiments on the mixtures of water ice with a complex organic mate-

\footnotetext{
* Corresponding author

Email address: bernhard.jost@space.unibe.ch (Bernhard Jost)
} 
rial (tholins) and carbonaceous compounds (carbon black; activated charcoal) studied in our companion publication (Jost et al., submitted). Sublimation experiments are needed to develop the particular texture (high porosity), expected on the nucleus' surface, which might have a strong influence on spectrophotometric properties. The spectrally best matching mixtures of non volatile organic molecules from Jost et al. (submitted) are mixed with fine grained water ice particles and evolved in a thermal vacuum chamber, in order to monitor the influence of the sublimation process on their spectro-photometric properties.

We demonstrate that the way the water ice and the non-volatile constituents are mixed, plays a major role in the formation and evolution of a surface residue mantle as well as having influence on the consolidation processes of the underlying ice. Additionally it results in different activity patterns under simulated insolation cycles. Further we show that the phase curves of samples having a porous surface mantle layer display higher coincidence with data of $67 \mathrm{P}$ than the phase curves of the samples having a more compact surface layer with smooth texture.

The analysis of spectral absorption bands of water ice in the near-infrared (NIR) range, similar to those acquired by the VIRTIS instrument onboard Rosetta, allows to link compositional considerations with surface activity and texture.

Keywords: Hyperspectral imaging, water ice, organics, comets

\section{Introduction}

Cometary surfaces are in permanent change through sublimation processes. The evolution of ices mixed with minerals and organic components is complex and requires laboratory constraint. Until today only a few laboratory studies 5 have been performed to investigate the sublimation of ices mixed with minerals and organic components, prepared as analogues of cometary nuclei. In the 
1970's simulation experiments were made at the Joffe Institute in Leningrad (Dobrovolsky and Kaimakov, 1977), mixing ice with minerals and/or organics. Later in 1980's a group at JPL in Pasadena carried out sublimation experiments of water ice samples prepared from suspensions with phyllosilicates, other silicates and organic matter (Saunders et al., 1986; Storrs et al., 1988). The most extensive and comprehensive campaign was the KOSI (KOmeten-SImulations) experiments performed at the DLR space simulator in Köln from 1987 to 1993 (e.g. Gruen et al., 1991; Gruen et al., 1993; Seiferlin et al., 1995). The focus of these experiments laid, similar to our own experiments, on the surface evolution and sublimation processes under simulated space conditions, but with larger scale samples. Although 11 experimental runs were performed, there was no systematic approach in mixing constituents. For the KOSI 3, 4 and 6 experiments, Oehler and Neukum (1991) measured the reflectance spectra between 0.36 and $2.5 \mu \mathrm{m}$ before and after the sublimation experiments under three different illumination angles.

The benefit of our recent experiments in Bern (Pommerol et al., 2015a; Poch et al., 2016a,b) is the possibility to acquire spectral data in-situ during the sublimation process through a window of the vacuum chamber. In this work a photometric characterization before and after the sublimation is added to investigate the bidirectional reflectance quantities.

In our companion paper (Jost et al., submitted) we performed a systematic laboratory study of potential cometary compositions in order to match the observed surface properties of $67 \mathrm{P}$. We measured the bidirectional reflectance of carbon-tholin-, carbon-water ice- and tholin-water ice-mixtures. It was possible to reproduce the individual spectrophotometric parameters (albedo, spectrum, phase reddening, phase curve) of $67 \mathrm{P}$, however no mixture was able to fit all 35 parameters alltogether.

We have indicated that the samples with the highest porosities best match 
the phase curve of $67 \mathrm{P}$. Spectral considerations show that the particle size of the darkening agent has a lower limit of a few hundred nanometres to several micrometres and cannot be dominated by particles in the range of some tens of nanometres. This finding is based on the blue spectral slope observed on nanometre-sized carbon black and the arch-shaped spectral curve when mixing carbon black with tholins.

Further our findings from Jost et al. (submitted) support the idea that the bright ice patches observed by OSIRIS and VIRTIS should be relatively dust free at small scale as already small dust contaminations are able to decrease the albedo dramatically. from the companion work and added fine grained water ice particles to investigate sublimation processes. The integrated VIS-NIR reflectance spectra of both carbon types (carbon black, activated charcoal) as well their mixtures with tholins from Jost et al. (submitted) are shown in Fig. 1. These two mixtures are the basis for the current work where a series of three individual sublimation experiments was performed using the same production protocol, but different compositions. Mixtures of fine grained spherical ice particles with carbonaceous compounds and complex organic matter were sublimed and spectroscopically investigated. We report on the acquired spectra in the $0.40-2.35 \mu \mathrm{m}$ range, resulting in a disappearance of water absorption bands as the ice sublimes and a residue layer accumulates. Further we characterise the spectral slopes and the visible reflectance during the sublimation process.

\section{Methods}

\subsection{SCITEAS}

All sublimation experiments were performed with the SCITEAS setup (Simulation Chamber for Imaging the Temporal Evolution of Analogue Samples). In this 


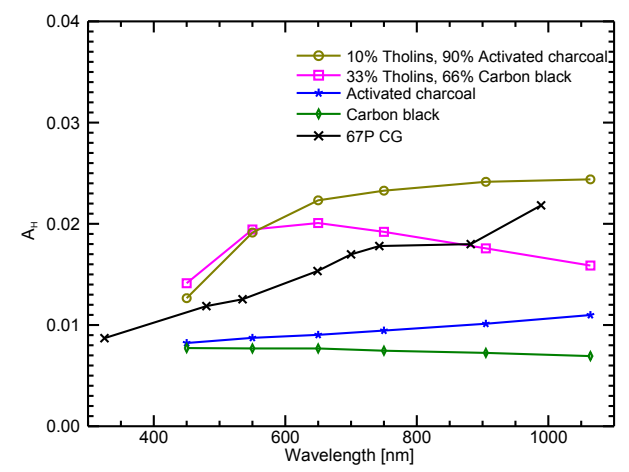

Figure 1: Integrated reflectance spectra of the non-volatile components as used for sublimation experiments in this work. The selection is based on the findings of Jost et al. (submitted).

paragraph only a short description is provided, more detailed information of this simulation chamber and its imaging system can be found in Pommerol et al. (2015a).

The SCITEAS thermal-vacuum chamber accommodates a cylindrical sample holder of $160 \mathrm{~mm}$ diameter that can be equipped with up to 4 small sample containers. A hyperspectral imaging system characterizes the reflected light from the sample in the 0.40 to $2.50 \mu \mathrm{m}$ spectral range through a $15 \mathrm{~mm}$-thick fused quartz window. The sample is illuminated from a monochromatic light source (halogen lamp coupled to a monochromator) through the window by an optical fibre. Two broadband cameras, VIS and NIR, placed at 200 and $300 \mathrm{~mm}$ above the window collect the scattered light at an angle of about $13^{\circ}$ with respect of nadir direction. The visible camera covers a spectral range of $0.38-1.08 \mu \mathrm{m}$, the near-infrared camera $0.85-2.50 \mu \mathrm{m}$. To create hyperspectral cubes the monochromatic light source shifts the wavelength through the spectral range (with a sampling of $6-20 \mathrm{~nm}$ ). At each wavelength an image is acquired.

The sample holder is radiatively cooled from a cylindrical shroud with contin85 uous liquid nitrogen circulation. The chamber is evacuated using two membrane primary pumps and one turbomolecular pump. The long term sample holder 
temperatures achieved with this cooling system lie in the -90 to $-75^{\circ} \mathrm{C}$ range, depending on the vertical distance from the window. The minimum pressure lies between $10^{-5}$ mbar and $10^{-6}$ mbar, depending on the content of volatiles.

A solar simulator (L.O.T. Quantum Design, product \# LS0308) with a $300 \mathrm{~W}$ Xe arc lamp yielding an intensity of 1 solar constant on a $40 \mathrm{~mm}$ diameter spot is placed above the window of the vacuum chamber to illuminate the samples.

During all three experiments the NIR data quality was strongly influenced by temperature fluctuations in the laboratory. The cycling operation mode of the ventilation and cooling system lead to variations of dark current of the sensor. This effect could be directly observed in the spectra as temporal fluctuations in reflectance, as the spectra are acquired as a time sequence, scanning the spectral range. We were able to compensate for this problem to some extent by analysing the signal within a non-illuminated area beside the sample holder, and correcting the dark level in the calibration. But some wave-like artefacts can still be seen on the spectra. Due to a very low albedo of our sample materials in these experiments, the signal to noise ratio was critically low during the first two experiments, especially in the NIR range. By optimising the exposure times and performing multiple acquisitions at each wavelength, which are averaged, it was possible to reduce spectral noise.

\subsection{PHIRE-2}

The bidirectional reflectance measurements presented in this work were acquired using the PHIRE-2 radio-goniometer. Details about the instrument and its calibration can be found in Pommerol et al. (2011) and Jost et al. (2016), therefore only basic specifications are summarized here. The goniometer is located inside a freezer, operated at $-35{ }^{\circ} \mathrm{C}$ for ice-bearing samples. Non-ice samples were measured at room temperature. A collimated light source is equipped with six bandpass filters $(450 / 550 / 650 / 750 / 905 / 1064 \mathrm{~nm})$ and illuminates the 
samples under an incidence angle of $0^{\circ}$ to $60^{\circ}$. The scattered light is measured with a silicon photovoltaic sensor on another mobile arm, the $-80^{\circ}$ to $+80^{\circ}$ emission angle range. The phase angle $\alpha$ is the angle between incidence and emission direction. In this work only the $750 \mathrm{~nm}$-filter was used in order to reduce the acquisition time to a minimum and prevent sublimation effects.

PHIRE-2 can be equipped with either a mirror head or a beam splitting system (Jost et al., 2016). The advantage of the beamsplitter is the possibility of measuring very low phase angles (down the $0^{\circ}$ ) compared to a mirror, which blocks the incoming light at phase angles smaller than $4^{\circ}$. However the signal is by a factor 4 lower with the beamsplitter than with the mirror. This plays no role for bright samples as studied in Jost et al. (2016), but is a serious issue in case of very dark samples as in this study.

\subsection{Sample material}

The sample compositions were selected in order to act as cometary analogues after significant sublimation. The basic constituent is fine grained ice produced by spraying water into liquid nitrogen. As composition of non-volatiles a combination of complex organic material and carbonaceous compounds was used. highest coincidence in spectrum and albedo with comet 67P (Fornasier et al., 2015). The amount of non-volatiles in our experiments $(0.3-2.0 \%)$ are by one to two orders of magnitude lower than in a comet's nucleus, but as we sublime the ice in the uppermost layers, the (photometrically relevant) ratio may 140 be comparable. To see effects of different spatial distributions of non-volatiles inside the ice matrix, two different mixing types were adopted.

All characteristics concerning sample composition and measurement configurations are listed up in Table 1. Experiment \#1 was performed using carbon black as dark compound, experiments \#2 and \#3 were prepared using activated charcoal. The mixing ratio of charcoal and tholins was defined by the closest 
match of this mixture compared to the visible spectrum of $67 \mathrm{P}$ (Jost et al., submitted). Experiment \#3 has a higher initial concentration of non-volatiles compared to \#2 (2\% instead of $0.3 \%$ ). The sublimation duration was chosen to have a relatively ice free residue layer with low surface irregularities.

Table 1: Characteristics of sublimation experiments.

\begin{tabular}{|c|c|c|c|}
\hline & Experiment \#1 & Experiment \#2 & Experiment \#3 \\
\hline Initial water ice content & $99.7 \mathrm{wt} \%(67 \pm 31 \mu \mathrm{m})$ & $99.7 \mathrm{wt} \%(67 \pm 31 \mu \mathrm{m})$ & 98.0 wt $\%(67 \pm 31 \mu \mathrm{m})$ \\
\hline Initial carbonaceous compound content & $0.2 \mathrm{wt} \%$ carbon black (42 $\mathrm{nm})$ & $0.27 \mathrm{wt} \%$ activated charcoal $(17.6 \pm 11.3 \mu \mathrm{m})$ & $1.8 \mathrm{wt} \%$ activated charcoal $(17.6 \pm 11.3 \mu \mathrm{m})$ \\
\hline Initial organics content & $0.1 \mathrm{wt} \%$ titan tholins $(315 \pm 185 \mathrm{~nm})$ & $0.03 \mathrm{wt} \%$ titan tholins $(315 \pm 185 \mathrm{~nm})$ & $0.2 \mathrm{wt} \%$ titan tholins $(315 \pm 185 \mathrm{~nm})$ \\
\hline \multirow[t]{3}{*}{ Sample types } & \multirow[t]{3}{*}{ intra + inter-mixture } & intra + inter-mixture & intra + inter-mixture \\
\hline & & \multirow[t]{2}{*}{ pure charcoal/tholins powder } & coarse fraction (> $800 \mu \mathrm{m}$ ) of intra-mixture \\
\hline & & & from sieving pure ice \\
\hline Bulk density & $0.4-0.5 \mathrm{~g} / \mathrm{cm}^{3}$ & $0.4-0.5 \mathrm{~g} / \mathrm{cm}^{3}$ & $0.4-0.5 \mathrm{~g} / \mathrm{cm}^{3}$ \\
\hline Sublimation duration before & \multirow[t]{2}{*}{$6.45 \mathrm{~h}$} & \multirow[t]{2}{*}{$14.25 \mathrm{~h}$} & \multirow[t]{2}{*}{$6.20 \mathrm{~h}$} \\
\hline 2nd goniometer measurement & & & \\
\hline Continuation after & \multirow[t]{3}{*}{ no } & \multirow[t]{3}{*}{ no } & $7.5 \mathrm{~h}$ normal sublimation \\
\hline \multirow[t]{2}{*}{ 2nd goniometer measurement } & & & afterwards day-night cycles \\
\hline & & & with solar simulator \\
\hline
\end{tabular}

\subsubsection{Ice particles}

The spherical water ice particles used in this study have a diameter of $67 \mu \mathrm{m}$ with a standard deviation of $31 \mu \mathrm{m}$. For production details see Yoldi et al. (2015).

\subsubsection{Tholins}

We used tholins as analogues of the complex organic matter, because they exhibit a strong red spectral slope from 0.2 to $1.0 \mu \mathrm{m}$, allowing us to vary the reddness and albedo of our samples by mixing it with darker and spectrally different carbonaceous compounds. The word "tholins" was first used by Sagan and Khare (1979) to describe the complex macromolecular organics formed after the irradiation of a gas mixture composed of simple molecules, displaying a orange-brownish colour. The yellowish to dark organic residues obtained after the irradiation of simple ices have also been named tholins or "ice tholins" (see e.g. McDonald et al., 1996). The tholins used in this work 
material was produced in a plasma of $95 \% \mathrm{~N}_{2}$ and $5 \% \mathrm{CH}_{4}$, using the PAMPRE setup (Sciamma-O'Brien et al., 2010; Szopa et al., 2006). Tholins are made of a complex mixture of molecular and macromolecular material composed of aliphatic hydrocarbons, nitriles, imines, amines or N-bearing aromatic compounds (Bernard et al., 2006; Carrasco et al., 2009; Pernot et al., 2010; Quirico et al., 2008; Gautier et al., 2014; Gautier et al., 2016). The complex organic molecules are arranged in high porosity spherical grains with a mean diameter of $315 \pm 185 \mathrm{~nm}$ (Carrasco et al., 2009). Their optical indices have been characterised by Mahjoub et al. (2012) and the reflectance spectra by de Bergh et al. (2008).

\subsubsection{Carbon powders}

We used carbon powders as analogues of the darkening agent(s) of cometary surfaces. Amorphous carbon inherited from interstellar dust could represent up to $10 \%$ of cometary materials (Wooden, 2008) and could partly contribute to the low albedo of cometary nuclei. However such a high amount of pure carbon in cometary refactory material is questionable, since by date only complex organic materials rather than pure carbons have been found in natural extraterrestrial samples like porous interplanetary dust particles (IDP) or STARDUST samples (e.g. Sandford et al., 2006; Brownlee, 2014).

Carbon black

The carbon black used in this work was purchased from Alfa-Aesar (product 3972). It is the solid residue obtained by acetylene combustion and consists of amorphous carbon shaped in spherical particles with an average diameter of $42 \mathrm{~nm}$. The structure of the individual particles cannot be resolved in Fig. 2a.

\section{Activated charcoal}

The activated charcoal used in this study was purchased from Sigma-Aldrich (Product C3345). This granular carbon has been prepared from wood particles which have been impregnated with a chemical agent and pyrolysed to produce very porous particles of pure carbon. The particles are smaller than $75 \mu \mathrm{m}$ 
according to the manufacturer. Our own microscope image analysis resulted in an average diameter of $17.6 \mu \mathrm{m}$ with a standard deviation of $11.3 \mu \mathrm{m}$. The particle shape is irregular with sharp edges (see Fig. 2b). There is very little formation of agglomerates.

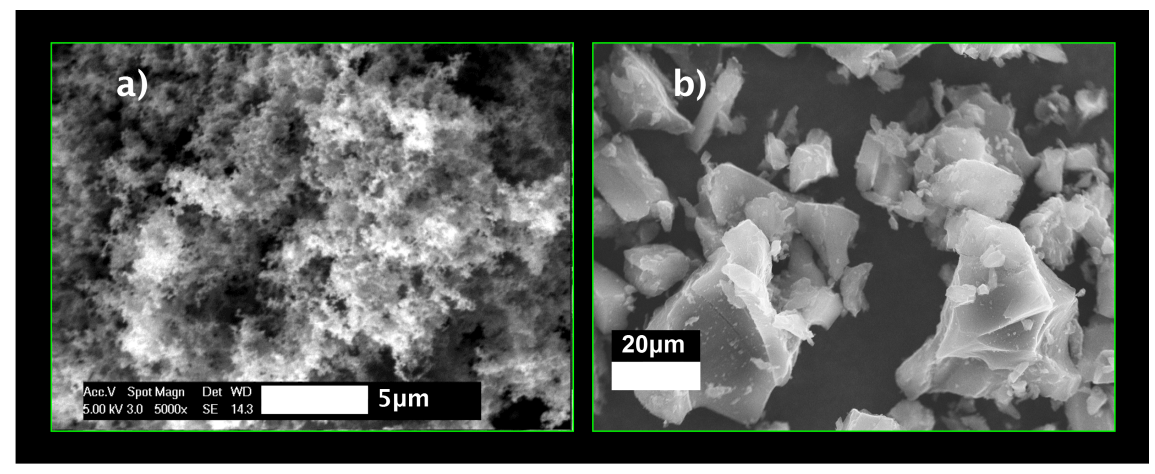

Figure 2: Environmental scanning electron microscope images of of carbon black (a) and activated charcoal (b) acquired at Institute of Geography at University of Bern.

\subsubsection{Mixing types}

200 laboratory analogues:

1. Intra-mixture, tholins and carbon particles are suspended in water prior to the freezing process. The non-volatiles are present as inclusions within the icy grains. The suspension is prepared by ultrasonication, performed with a Hielscher 200Ht ultrasonic unit equipped with a $7 \mathrm{~mm}$ diameter sonotrode. The duration of ultrasonication was limited to about 1 min to keep the suspension temperature below $30^{\circ} \mathrm{C}$, to avoid thermal changes.

2. Inter-mixture, non-volatiles are mixed intimately with the pure water ice particles at the grain level. The mixture was prepared in an aluminium bottle cooled to nitrogen temperature, by adding freshly produced ice and precooled non-volatiles. The bottle was then agitated using a VortexGenie2 mixer at full speed for about $1 \mathrm{~min}$, interrupted by re-cooling the bottle in liquid nitrogen. 


\subsubsection{Sample preparation} 20x40x20 mm. The deposition of sample material into the sample holder was done by sieving $(800 \mu \mathrm{m}$ mesh size) to avoid big agglomerates and to produce homogeneous samples of controlled bulk density. There is no compression, the piled material is cut with a spatula and a thin layer of material is sieved on top to obtain a random surface orientation. In experiment \#3 the remaining material from the sieving process was used as a separate sample (coarse-grained intra-mixture) with a very granular structure.

\subsubsection{Measurement sequence}

After sample production and preparation in the compartments the intraand inter-mixtures samples were measured in the PHIRE-2 goniometer individually, taking $40 \mathrm{~min}$ each. The other sample was stored under a cover in a chest freezer at $-40^{\circ} \mathrm{C}$. After the goniometer data acquisition the compartments were arranged in a bigger sample holder, pre-cooled to liquid nitrogen temperature, fitting into the vacuum chamber.

230

After the sample holder was placed into the SCITEAS chamber and the chamber was closed, the liquid nitrogen cooling cycle was started. After some minutes the primary and the secondary vacuum pumps were started stepwise, reaching a final pressure of $10^{-5}$ mbar after $\sim 30 \mathrm{~min}$. When the cooling stabi235 lized $\sim 1 \mathrm{~h}$ after the beginning, the ambient light in the laboratory was turned off in order to acquire a first hyperspectral cube.

The aim of these sublimation experiments was to create a compact, flat residue layer of non-volatiles on the samples. However we wanted to avoid ejections of these layers (as observed in previous experiments), to keep a smooth texture in order to be able to acquire a second BRDF dataset with the goniometer after some hours. 
Experiments \#1 and \#3 had sublimation durations of $6.45 \mathrm{~h}$, and $6.20 \mathrm{~h}$ due to a higher initial brightness.

The samples from the experiment \#3 were re-inserted into SCITEAS after the second measurement in PHIRE-2 and left to sublime during additional $7.5 \mathrm{~h}$. Afterwards a sequence of 3 illumination cycles with the solar simulator was run (20 min illumination, $20 \mathrm{~min}$ dark). Due to the $40 \mathrm{~mm}$ diameter illumination field, all samples are only illuminated partially, some areas stay always in the shadow (see Fig. 7).

\subsection{Spectrophotometry and surface morphology}

3.1.1. Experiment \#1: 99.7\% water ice $+0.2 \%$ carbon black $+0.1 \%$ tholins

For experiment \#1 ice grains were prepared as inter- and intra-mixtures using a powder of non-volatile materials made of $33 \%$ tholins and $67 \%$ carbon black and sublimated during $6.45 \mathrm{~h}$. The integrated reflectance spectrum (hemispherical albedo) of the dry powder acquired with PHIRE-2 can be found in Fig. 1. It shows a steep red slope from 450 to $650 \mathrm{~nm}$ and a shallow blue slope towards the NIR.

The freshly deposited inter-mixture sample (Fig. 3a) has a greyish tone, the intra-mixture is slightly darker and less yellowish. The middle of the sample is covered with frost streak deposited from air flow above the surface at the 
beginning of the experiment prior to the start of the vacuum pumps. After $15 \mathrm{~min}$ the frost layer is sublimated and the inter-mixture displays a yellowish tone (Fig. 3b). The intra-mixture is still darker with coverage of black ice grains on the surface. Two hours after the start the intra-mixture formed an uniform, black residue layer (Fig. 3c), while the inter-mixture is not as yellowish as before and has some transparent ice grains on the surface. It took about $4 \mathrm{~h}$ until the inter-mixture had a visually similar smooth residue layer as the intra-mixture, with a slight yellowish tone and no ice visible.

The reflectance spectrum in the $0.40-2.35 \mu \mathrm{m}$ range acquired with the SCITEAS hyperspectral imaging system can be found in Fig. 4. Fig. 4a compares the spectra of the two mixture types after $1 \mathrm{~h}$ of sublimation. The intramixture is about $50-70 \%$ darker and more linear red compared to a concave shape of the inter-mixture below $1 \mu \mathrm{m}$. The spectra are featureless in the visible. Data up to $0.94 \mu \mathrm{m}$ is acquired with the visible camera and less noisy due the higher signal. The NIR spectra clearly show the two water ice absorption bands at $1.5 \mu \mathrm{m}$ and $2.0 \mu \mathrm{m}$; the bands of the inter-mixture are a bit more pronounced and the spectra less noisy.

The temporal evolution of the reflectance spectrum of the inter-mixture sample is shown in Fig. 4b, the reflectance in the visible drops by almost $100 \%$ after $3.10 \mathrm{~h}$ compared to the measurement after $1 \mathrm{~h}$ and the water absorption bands start to disappear. At the band center of the $2.0 \mu \mathrm{m}$ band the reflectance increases by a factor of 2 between the first and the last spectrum.

\subsubsection{Experiment \#2: $99.7 \%$ water ice $+0.27 \%$ charcoal $+0.03 \%$ tholins}

In experiment \#2 a mixture of $10 \%$ tholins and $90 \%$ activated charcoal was used to mix with ice. The PHIRE-2 integrated spectrum of thispowder can be found in Fig. 1, it is monotonically red with a steeper slope in 450 to $650 \mathrm{~nm}$ range originating from the tholins. Compared to experiment \#1 where nanometre-sized carbon black was used as darkening agent, experiment \#2 con- 


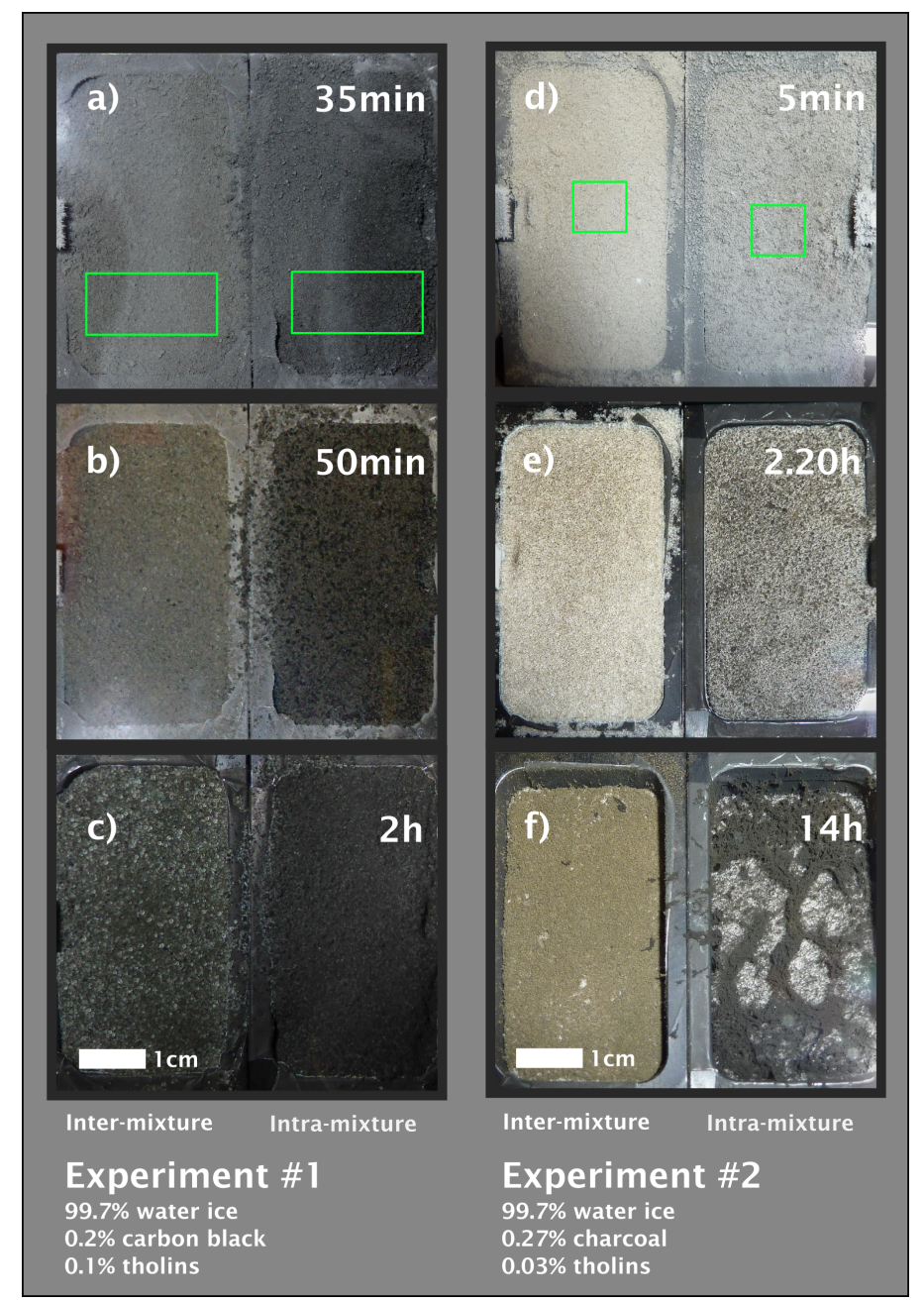

Figure 3: Time sequences of sublimation experiments \#1 (a-c) and \#2 (d-f). The intramixture sample is always positioned on the right side, the inter-mixture on the left. The green boxes indicate the regions of interest (ROI) for spectral analysis. The streaks in a) originate from frost deposit at the beginning of the experiment and have sublimed in $b$ ). The intra-mixture in b) shows some dark grains in the top layer while the inter-mixture appears still uniform. After 2 h (c) the inter-mixture shows transparent ice grains on the surface while the intra-mixture has an uniform residue layer of dark material. Experiment \#2 displays a significant yellowish colour originating from tholins. Overall the sample is brighter than experiment \#1 due to bigger carbon particles, besides the identical mixing ratios. Again the intra-mixture has a more heterogeneous colour, with some dark grains on the surface. After $14 \mathrm{~h}$ (f) the inter mixture shows an almost uniform texture, the intra-mixture experienced some gas driven ejection events, creating a filamentous matrix with a heterogeneous texture with exposed ice. 

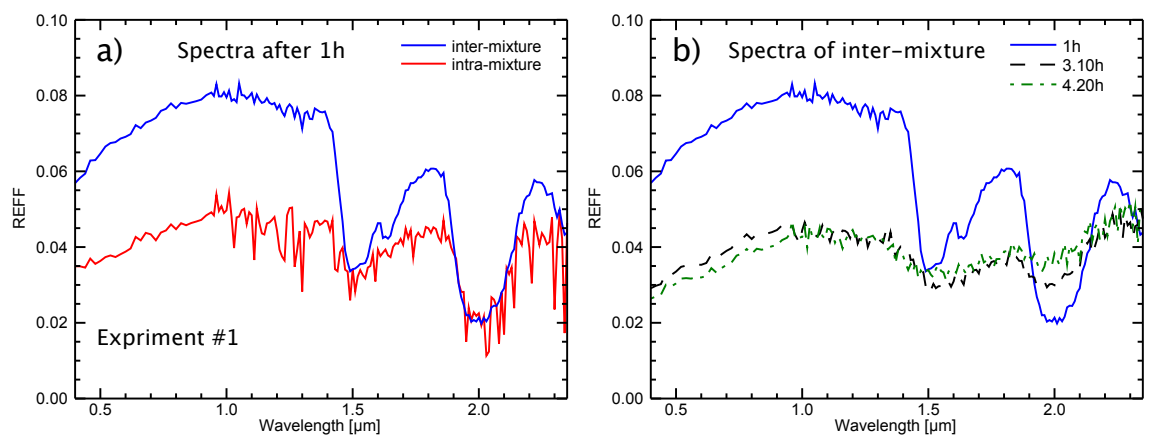

Figure 4: Reflectance spectra from experiment \#1 in the $0.4-2.35 \mu \mathrm{m}$ range. a) comparison of the mixture types after $1 \mathrm{~h}$ of sublimation. The intra-mixture is about $50-70 \%$ darker in the visible spectral range and up to about $1.4 \mu \mathrm{m}$, the reflectance in the water absorption bands at $1.5 \mu \mathrm{m}$ and $2.0 \mu \mathrm{m}$ is almost identical. b) temporal evolution of spectra from the inter-mixture. The sample becomes darker by nearly $100 \%$ after $3.10 \mathrm{~h}$. The water absorption bands start to vanish but are still present. At $2.0 \mu \mathrm{m}$ the sample increases in reflectance after 4.20 h by nearly $100 \%$ compared to the first spectrum.

tains micrometre-sized activated charcoal with an intrinsic red spectrum . Using a similar mass percentage of activated charcoal as of carbon black in the ice mixture increases the albedo, as the surface-to-mass ratio and therefore the total absorbing surface of bigger grains is lower. Thus samples of experiment \#2 are significantly brighter than the ones of experiment \#1 (Fig. 3).

The freshly deposited samples of experiment \#2 are marginally covered by frost (Fig. 3d), the intra-mixture appears greyish, the inter-mixture yellowish. After $2.20 \mathrm{~h}$ (Fig. 3e) the surface of the inter-mixture look still homogeneous whereas the intra-mixture starts to display heterogeneities with some darker grains on the top layer. On the last step in the time sequence after $14 \mathrm{~h}$, both samples have lost about $3-4 \mathrm{~mm}$ of material by sublimation (Fig. 3f). The inter-mixture has a yellowish-greyish colour with a smooth texture not showing any sign of ejection activity. The colour of the intra-mixture is dark-grey. The surface residue, forming a filamentous matrix with a heterogeneous texture is created by ejection events. These events are triggered by the upstreaming gas 
which breaks and ejects parts of the residue layer. The bright underlying material, still containing large amounts of ice, gets exposed to the surface.
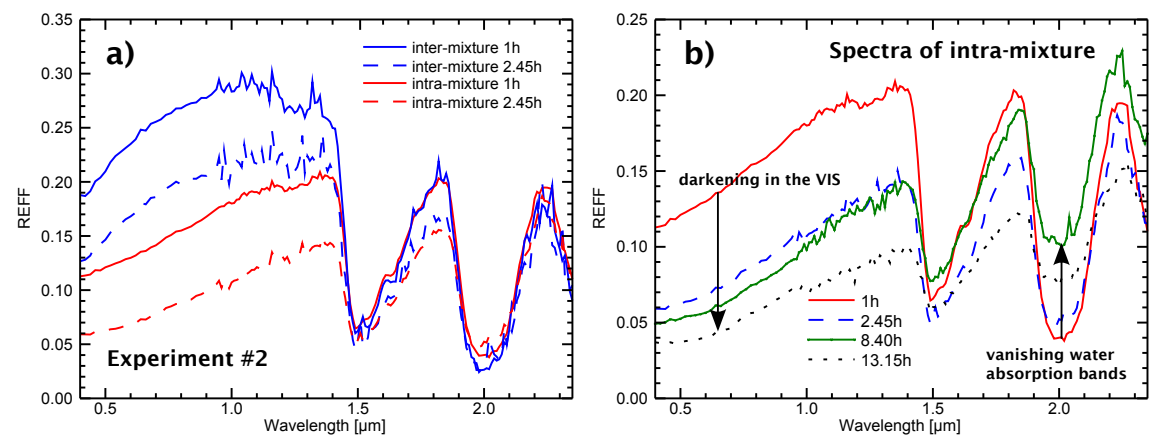

Figure 5: a) Reflectance spectra from experiment \#2 after 1 and $2.45 \mathrm{~h}$. Both samples become darker by about $50-80 \%$ in the visible range, in the infrared the spectrum remains almost constant except at around $1.8 \mu \mathrm{m}$. The red slope in the visible is more linear for the intramixture, the spectrum of the inter-mixture has a concave shape. The same phenomenon is visible in experiment \#1 (Fig. 4). b) Temporal evolution of the reflectance spectrum of the intra-mixture sample. There is no change in shape of the spectra in the visible range with time. The sample becomes darker with time in the visible, in the NIR the reflectance increases inside the water bands $(1.5 / 2.0 \mu \mathrm{m})$ and decreases on the inter-bands $(1.8 / 2.2 \mu \mathrm{m})$.

Reflectance spectra of experiment \#2 are plotted in Fig. 5. In Fig. 5a spectra of both mixing-types are compared at two times. Again the intra-mixture is generally darker and has a more linear spectrum in the visible range than the inter-mixture which is concave shaped. The NIR spectrum of the mixingtypes are nearly identical. After $1.45 \mathrm{~h}$ of sublimation both samples decrease in reflectance in the visible by $50-80 \%$ but the shapes of the spectra remain the same. In the NIR the reflectance at the centres of the water ice bands stays almost the same, but is decreased at the local reflectance maximum at at $1.8 \mu \mathrm{m}$. An extended time sequence (Fig. 5b) of the mixture up to $13.15 \mathrm{~h}$ displays a steady decrease in reflectance in the VIS with a constant slope angle and vanishing water absorption bands in the NIR, but the bands remain always visible. 


\subsubsection{Experiment \#3: $98 \%$ water ice $+1.8 \%$ charcoal $+0.2 \%$ tholins}

335 amount of contaminants versus water ice (2\% instead of $0.3 \%$ ) which makes the samples darker. A time sequence showing the evolution of the samples is provided in Fig. 6. In additional to two mixing-types (inter/intra) a sample of pure ice and a sample with the remains from the sieving process of the intra-mixture are added. The remaining material from the sieving is made of agglomerates larger than $800 \mu \mathrm{m}$ and appears much darker than the fine fraction obtained after the sieving process. Compared to experiments \#1 and \#2 the inter-mixture does not appear as yellowish. After $1 \mathrm{~h}$ (Fig. 6a) of sublimation the intra-mixture looks marginally darker with some dark grains on the surface, to a thin frost layer.

Fig. 6c shows the samples after the first 20 min illumination cycle with the solar simulator at 1 solar constant. The position of the light spot can be seen 355 in Fig. 7. Already after 2 min of illumination a cm-sized patch of the intramixture was ejected and the underlying material was exposed. The ejecta of the inter-mixture are mm-sized and started only after $8 \mathrm{~min}$. The later beginning indicates either a higher gas permeability or lower sublimation rate, the smaller patches a lower cohesion force. This is consistent with findings made

and ejection patches, only individual grains are redistributed across the surface. The pure ice shows no textural changes except that a buried temperature sensor is exposed and some ejected particles from the other samples are deposited. 


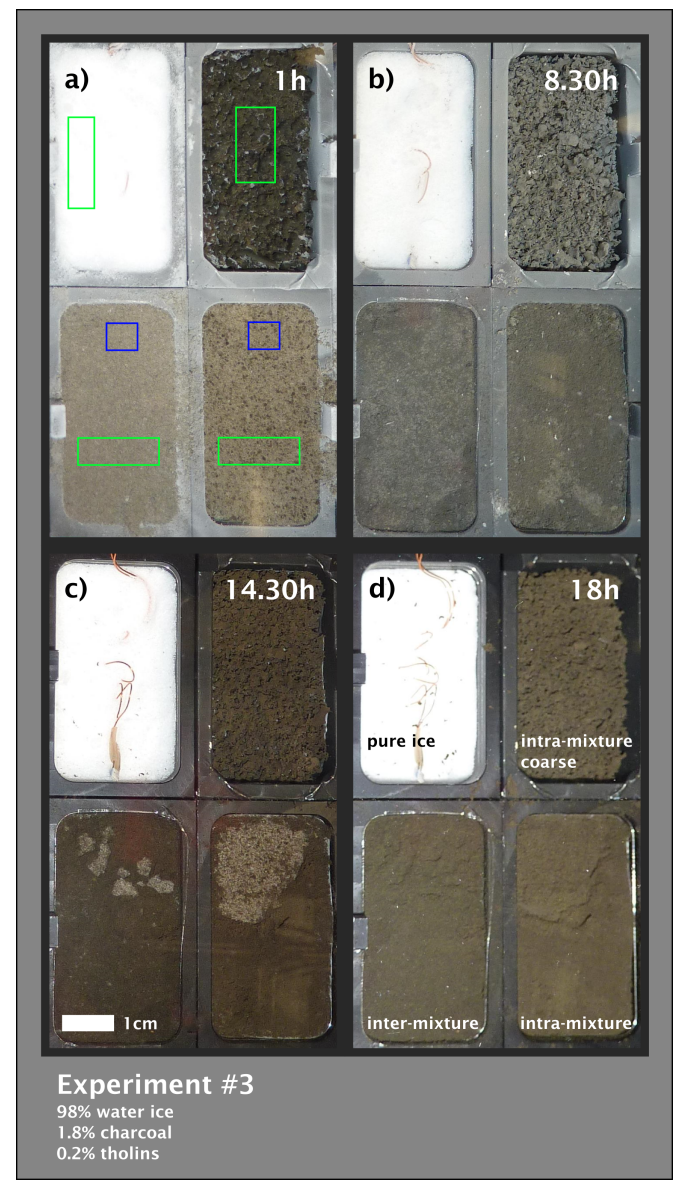

Figure 6: Time sequences of sublimation experiment \#3. The ROI used for spectral analysis are indicated in green, the ones of the illuminated area in blue. b) shows the samples after the measurement with the goniometer, where the vacuum chamber had to be opened, the samples removed and inserted again. This explains the re-deposition of frost that hides the reddish colour. c) shows the samples after 20 min of illumination with a 1 solar constant solar simulator in the central part (see Fig. 7). Inside the illuminated area of the intra- and inter-mixture the residue layers are ejected and fresh sample material is exposed. The $\sim 1.5 \mathrm{~cm}^{2}$ residue patch of the intra-mixture was ejected after $2 \mathrm{~min}$ of illumination in one piece. The small patches of the inter-mixture lifted-off continuously after 8 min duration. After several cycles of illumination the exposed bright patches are re-surfaced and visually indistinguishable from the non-illuminated areas. The coarse fraction of the intra-mixture formed no solid residue layer, only individual grains are ejected and re-distributed, the granular texture stays visible until the end of the experiment. The pure ice displays no significant change in texture or ejection of grains. The temperature sensor gets exposed with time by mass loss and some dark grains from the other samples are deposited onto the surface. 
Compared to the dark mixtures the pure ice has a higher sublimation rate. During $14.30 \mathrm{~h}$ the pure ice pure ice sample lost about $4 \mathrm{~mm}$ of material compared to $\sim 2 \mathrm{~mm}$ on dark samples.

Fig. $6 \mathrm{~d}$ shows the samples at the very end of the experiment after several illumination cycles. All samples appear a bit brighter than $3.30 \mathrm{~h}$ before, this 370 is very likely an exposure/illumination effect as the copper wire and the anodized aluminium are also brighter. The mass loss of the pure ice sample is now $\sim 6 \mathrm{~mm}$, the intra- and inter-mixtures lost about $3 \mathrm{~mm}$ with a clear gradient towards the illuminated areas where more energy has been deposited. The pure ice shows no gradient as it is almost transparent in the spectral range of the 375 solar simulator.

All patches on which ejection events occurred, have been re-surfaced by a dry mantle and are indistinguishable by eye in terms of colour from the nonilluminated areas. Due to the ejection events the texture became more rough in the illuminated areas. The coarse grained sample shows no textural changes throughout the entire experiment.

In Fig. 8a the first spectra after $1.10 \mathrm{~h}$ of the three dark samples are compared. The inter-mixture is $\sim 20 \%$ brighter than the intra-mixture in the VIS, 385 both having a similar spectral shapes. The coarse grained intra-mixture is darker by a factor of 3 . In the NIR range the intra- and inter-mixture display a comparable water absorption band depth, while the spectrum of the coarse grained sample shows less deep water ice absorption bands. After $17.35 \mathrm{~h}$ of sublimation (Fig. 8b) all samples show a monotonically red spectrum which is almost identical in the VIS and slight variations in the NIR where signal noise is higher. There is no signal of water ice left anymore. These three spectra are all from non-illuminated areas of the samples. 


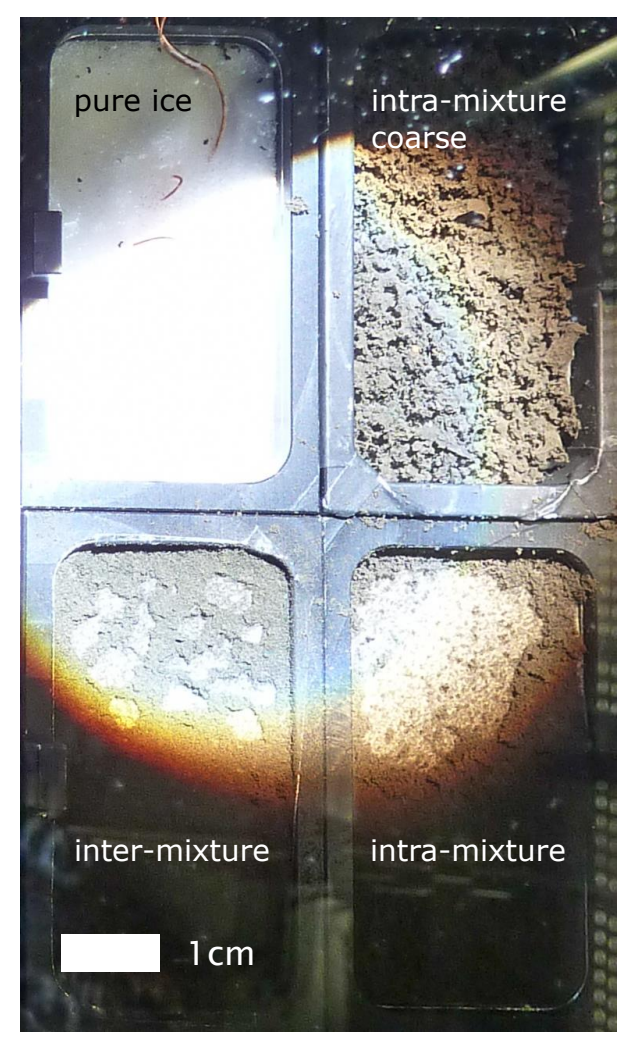

Figure 7: Experiment \#3 during the 2nd illumination cycle. It is obvious that the residue layer is only ejected inside the illuminated areas, the shape of the intra-mixture patch follows exactly the spot geometry. The intra-mixture (lower left sample) residue layer is more cohesive than the one from the inter-mixture. The coarse fraction of intra-mixture shows no structural dichotomy between the illuminated and shadowed areas. 

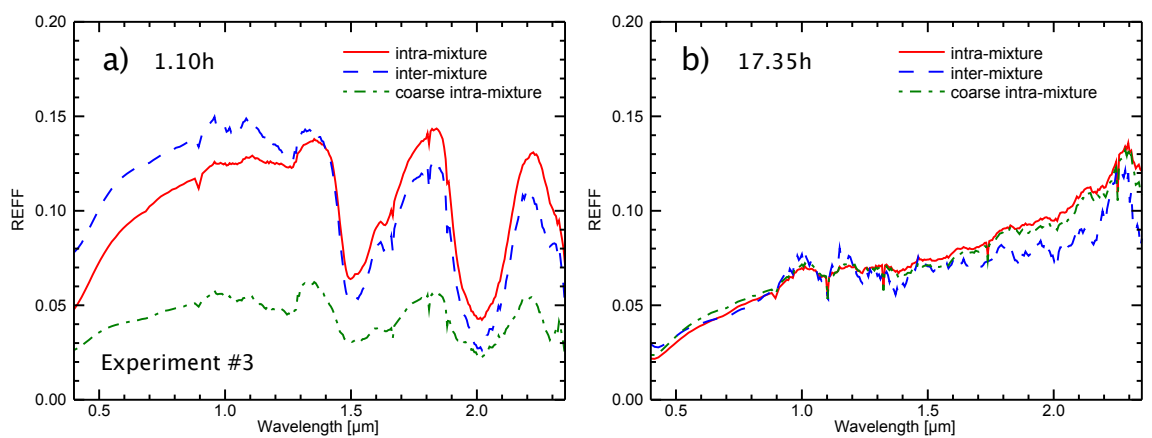

Figure 8: Reflectance spectra of all charcoal/tholin-bearing samples from experiment \#3. a) first spectra after $1.10 \mathrm{~h}$, the coarse fraction of intra-mixture is considerably darker throughout the entire spectral range, with less signature of water. The inter-mixture is about $20 \%$ brighter than the intra-mixture in the visible and the local maxima at 1.8/2.2 $\mu \mathrm{m}$ ) show lower reflectance values. b) Spectra of the non-illuminated areas from all samples at the end of the experiment. All samples display an almost identical spectrum without any hints of water ice in the NIR. The spectra are monotonically red with some instrumental artefacts clearly visible.

Fig. 9 shows spectra of the pure ice sample from experiment \#3, measured at three different times. The analysed area on the sample was selected so that the outstanding temperature sensor was not incorporated. The spectra show only a minor darkening in the VIS range within the first $4 \mathrm{~h}$ where no ejecta from other samples was deposited. After $17.35 \mathrm{~h}$ the spectrum became darker by $\sim 25 \%$ at $0.5 \mu \mathrm{m}$ with a red slope towards $1.0 \mu \mathrm{m}$. In the NIR spectral range the three spectra are indistinguishable to the level of instrumental errors. Four water ice absorption bands at 1.05/1.25/1.51/2.02 $\mu \mathrm{m}$ are clearly visible.

The fact that the NIR spectrum remains unchanged with a handful of very small grains inside the analysed area on the sample, but the VIS spectrum becomes darker and redder, leads to the conclusion that very fine grained tholin particles must have been deposited onto the surface of pure ice, invisible by eye. The fact that tholins have a very high albedo in the NIR would explain the unchanged spectrum in this region. This tholin powder could have been separated from the much bigger charcoal grains during the ejection events, or 
have been lifted off separately by gas flow from deeper layers of the samples.

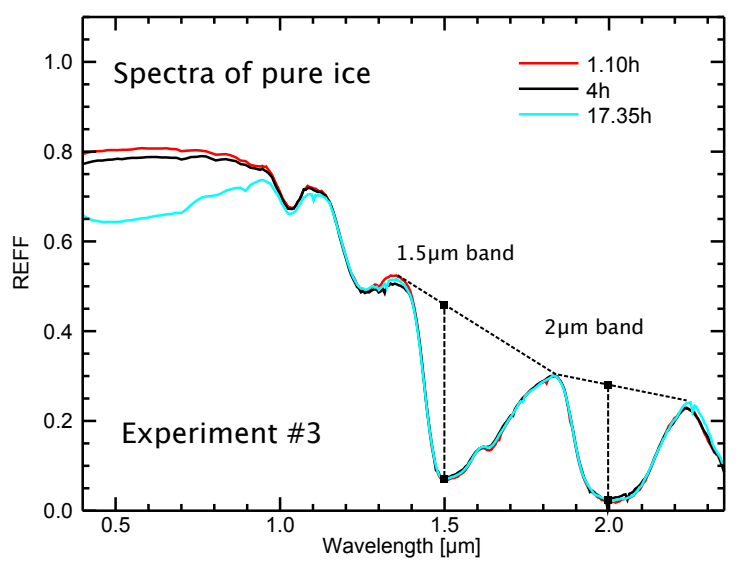

Figure 9: Spectra of the pure-ice sample from experiment \#3. In the visible range the spectrum after $17.35 \mathrm{~h}$ is darker and redder due to the deposition of ejected charcoal/tholinparticles from the other samples. In the NIR the spectrum shows no changes with time, there is no obvious effect of particle size changes (see Fig. 1.2 in Clark et al., 2013) due to sintering effects. The calculation of band depths at $1.5 / 2.0 \mu \mathrm{m}$ is illustrated. The two water ice absorption bands at 1.05/1.25 $\mu \mathrm{m}$ are clearly recognizable on the pure ice spectrum but are too weak to be distinguished on dark samples with lower signal-noise ratios. For definition of absorption bands see Tab. 11.1 in Mastrapa et al., 2013.

The spectra of illuminated and non-illuminated areas across the surface of the inter-mixture at the end of experiment $\# 3$ are compared in Fig. 10a. The illuminated area which was re-surfaced and has a rougher texture (see Fig. 6d) 415 is $\sim 20 \%$ brighter in the VIS up to $1.4 \mu \mathrm{m}$. The non-illuminated area shows a monotonically red spectrum in the NIR while the re-surfaced area shows features of the two most prominent water absorption bands at 1.5 and $2.0 \mu \mathrm{m}$. This becomes more pronounced when plotting the ratio of the two spectra: the non-illuminated spectrum is divided by the illuminated one (Fig. 10b). The deepening of bands at 1.5 and $2.0 \mu \mathrm{m}$ and enhancement of inter-bands at 1.8 and $2.2 \mu \mathrm{m}$ are clearly visible. 

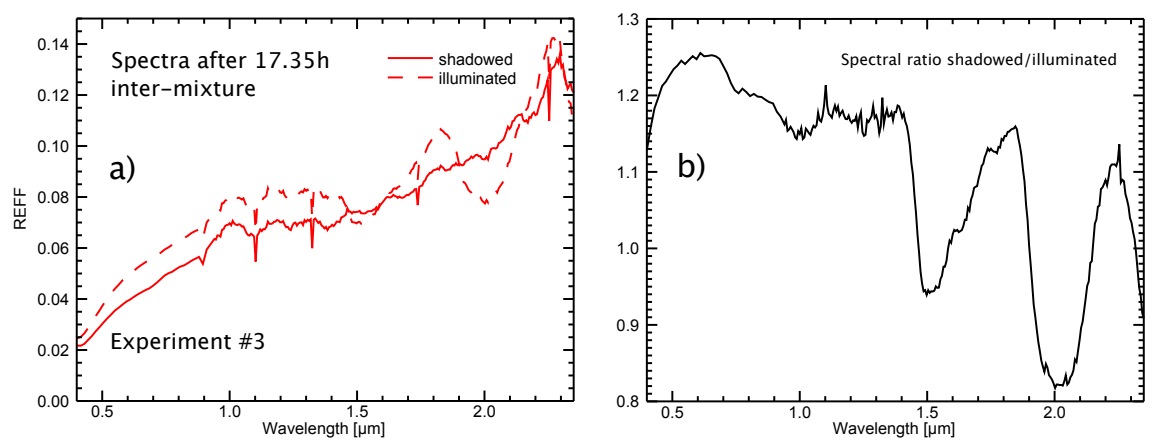

Figure 10: a) Spectral comparison of the illuminated (and re-surfaced) and the non-illuminated area. The re-surfaced area is $\sim 20 \%$ brighter in the visible with a similar red slope and shows signatures of water ice. b) spectral ratio between the two areas: values $>1$ mean that the illuminated area is brighter, values $<1$ mean darker than the non-illuminated area. The water ice absorption bands become clearly recognizable in the NIR, indicating a water ice content in the re-surfaced area.

\subsection{Bidirectional reflectance}

The principal goal of these sublimation experiments was to acquire and compare BRDF before and after the sublimation process. In order to analyse only the photometric changes of the surface mantle and not being influenced by ejection events, the sublimation duration had to be kept short to avoid ejection events to occur on the mantle, but long enough to create a porous layer. This was achieved on all samples except the intra-mixture from experiment \#2 (Fig. 3f). The BRDF-data from experiment \#1 was already analysed and compared to 67P in Feller et al. (2016).

The phase curved acquired at $0^{\circ}$ incidence angle and the $750 \mathrm{~nm}$ filter are plotted in Fig. 11, a) and c) show the two mixture types in absolute REFF, subfigures b) and d) show the same phase curves but normalized at $5^{\circ}$ phase angle, to better see the curvatures. In all subfigures the phase curve of comet 67P/Churyumov-Gerasimenko, derived from Hapke parameters at $743 \mathrm{~nm}$ from Fornasier et al. (2015) (Tab. 3 therein), are overlaid. The applied Hapke model in Fornasier et al. (2015) has 5 parameters (single term Henyey-Greenstein func- 

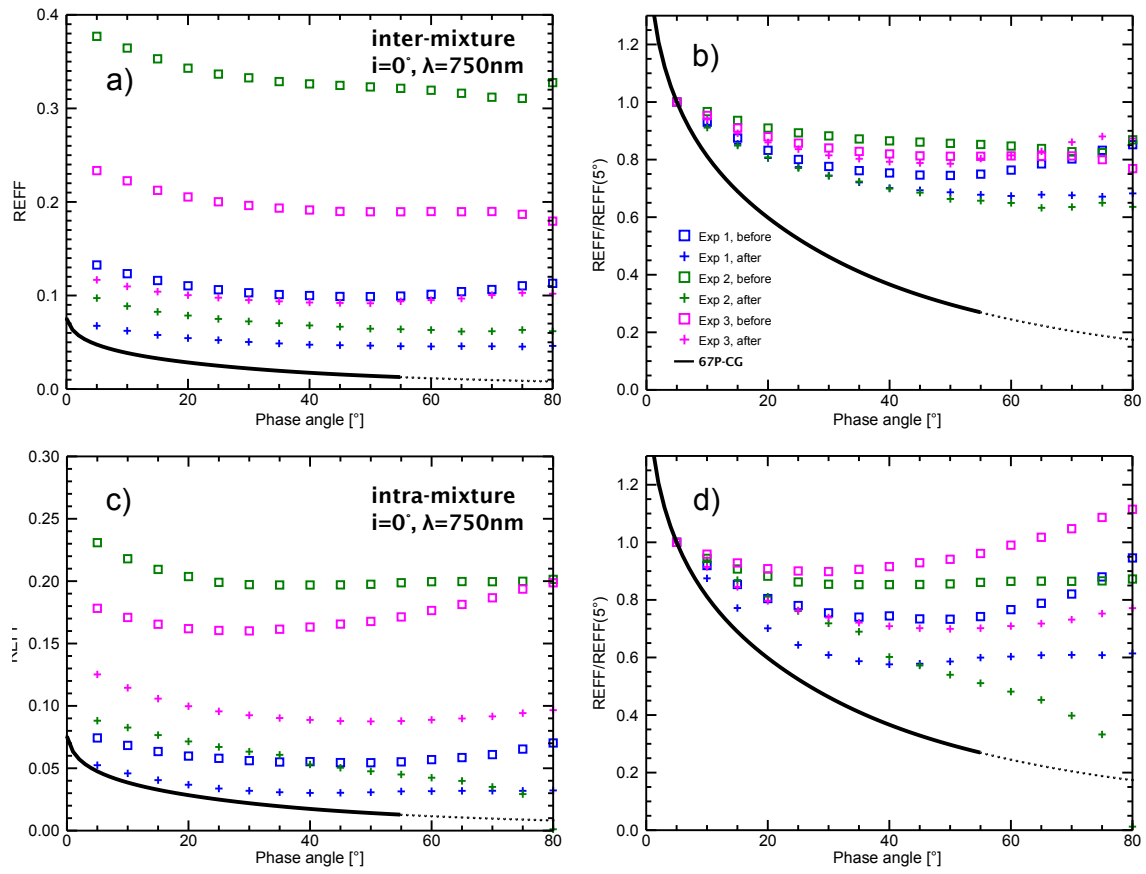

Figure 11: Phase curves for all experiments measured at $\mathrm{i}=0^{\circ}$ and $\lambda=750 \mathrm{~nm}$ before and after sublimation. a) Inter-mixtures in absolute reflectance, b) normalized at $\alpha=5^{\circ}$, c) intra-mixtures in absolute reflectance, d) intra-mixtures normalized at $\alpha=5^{\circ}$. All samples decrease in reflectance after sublimation in thermal vacuum. The intra-mixtures display a higher variation in shape when normalized than the inter-mixture. After sublimation the intra-mixtures have lower reflectances at high phase angles, which can be explained with a higher surface roughness or porosity. 
tion, no coherent-backscattering mechanism) and is derived from disk-averaged reflectance data.

The intra-mixture of experiment \#3 before sublimation is the only sample having the highest reflectance factor at high phase angle, the initial intramixture of experiment \#2 shows an almost flat phase curve from 30 to $80^{\circ}$ phase angle, \#1 is as well $\mathrm{u}$-shaped having almost the same REFF at $\alpha=5^{\circ}$ and $80^{\circ}$. The phase curves of the inter-mixtures before sublimation of experiments \#2 and \#3 are monotonically decreasing, the one from \#1 has a similar u-shape as the corresponding intra-mixture.

When analysing the normalized data the intra-mixtures show bigger changes in shape of the phase curves by sublimation than the inter-mixtures. The normalized reflectance at $\alpha=80^{\circ}$ of the inter-mixtures decreases only by $20 \%$ during the sublimation process of experiments \#1 and \#2, experiment \#3 shows nearly no variation. The corresponding intra-mixtures show bigger variations at this phase angle: Reflectance measured in experiments \#1 and \#3 decrease by $60-70 \%$, reflectance measured in experiment \#2 with its rough, filamentous surface structure (see Fig. 3f) decreases by more than a factor of 2 . This curve shows the highest coincidence with the cometary phase curve, which displays even lower relative reflectance at all phase angles, thought to originate from a very porous surface structure of organics, silicates, other opaque minerals, and carbonaceous compounds (Jost et al., submitted; Feller et al., 2016).

Fig. 12 shows the same selection of phase curves as in Fig. 11, but measured at $\mathrm{i}=60^{\circ}$. Compared to vertical illumination, influences of macroscopic surface roughness becomes more visible at higher incidence angles, where shadow cast can occur. All samples are significantly scattering in the forward direction (towards large phase angle), which is normally attributed to samples with low surface roughness (see Fig. 14 in Jost et al., 2013 for pure ice samples with different preparation methods). When normalized all samples have a higher 
for the intra-mixtures than for the inter-mixtures. The calculated phase curve of $67 \mathrm{P}$ is strongly less forward scattering than our samples, nevertheless is has to be considered that the maximal phase angle achieved from OSIRIS data and used to fit the Hapke model was about $55^{\circ}$. Everything beyond is extrapolated. Further, which can not be represented from cm-sized laboratory samples.
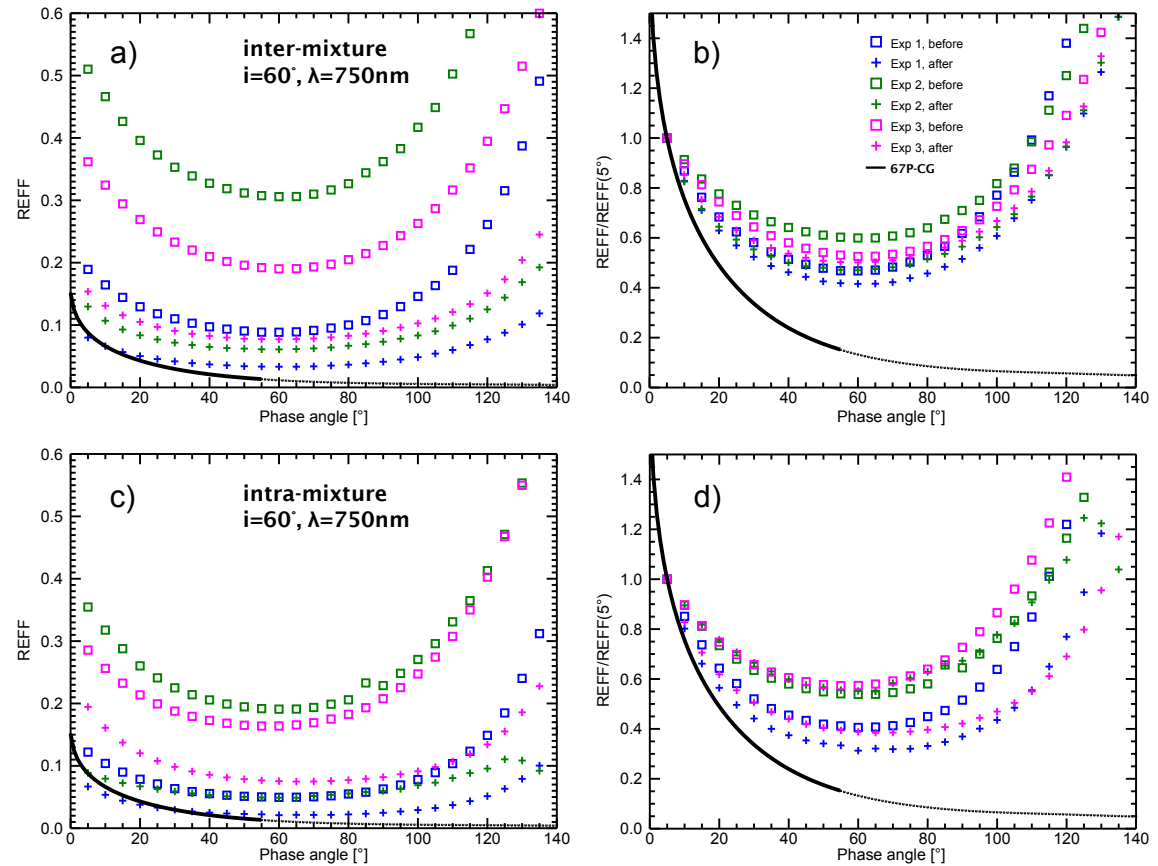

Figure 12: Phase curves of all experiments measured at $\mathrm{i}=60^{\circ}$ and $\lambda=750 \mathrm{~nm}$ before and after sublimation. a) Inter-mixtures in absolute reflectance, b) normalized at $\alpha=5^{\circ}$, c) intramixtures in absolute reflectance, d) normalized at $\alpha=5^{\circ}$. All samples decrease in reflectance after sublimation in thermal vacuum and become less scattering in the forward direction. The intra-mixtures display a higher variation in shape when normalized than the inter-mixtures. After sublimation the intra-mixtures have lower reflectances at high phase angles, which is consistent with a higher surface roughness or porosity.

All phase curves shown in this work are publicly available on the Data Analysis Center for Exoplanets (DACE) platform (https://dace.unige.ch/lossy/ 
samplesearch/index) where they can be freely visualized and downloaded. 


\section{Discussion}

In this section several fundamental characteristics such as brightness evolution, spectral slope and depth of absorption bands are discussed comparing the different experiments and samples. Further textural and structural evolution of experiment \#3 is analysed in detail.

485

In order to study the evolution of brightness in the VIS spectral range, the reflectance of all experiments at $750 \mathrm{~nm}$ is plotted as a function of time in Fig. 13. Experiment \#2 has the most complete dataset with the best temporal resolution. The reflectance of its inter-mixture seems to decrease exponentially during 13 hours. The intra-mixture shows the same trend during the first 5 hours, then slightly increasing in brightness due to break-up of the residue matrix, exposing bright material at the surface. Afterwards it becomes re-surfaced and decreases in reflectance until the end of the experiment. The intra-mixture remains always darker than the inter-mixture. Experiment \#1 shows a similar behaviour, the reflectance decreases non-linearly during $4 \mathrm{~h}$, the intra-mixture is darker by $\sim 50 \%$ in the beginning then the two converge towards similar reflectances. The decrease of experiment \#3 follows a similar trend, after $4 \mathrm{~h}$ the inter-mixture is minimally brighter, then the reflectances of the two curves converge.

When analysing the reflectance from the phase curves in Fig. 11, just after the initial sample preparation experiment \#2 is the brightest, followed by \#3 and \#1. After sublimating in vacuum for several hours (see Table 1) samples from experiment \#1 are the darkest, the ones from \#3 the brightest, but the absolute differences in reflectance became smaller after sublimation. An unknown parameter on all experiments is the amount of trapped frost on samples surfaces during the BRDF acquisition. The sample holders were at a temperature of about $-80{ }^{\circ} \mathrm{C}$ when taken out from the SCITEAS chamber and then inserted to the PHIRE- 2 freezer being operated at $-35{ }^{\circ} \mathrm{C}$ and $\sim 50 \%$ of relative hu- 


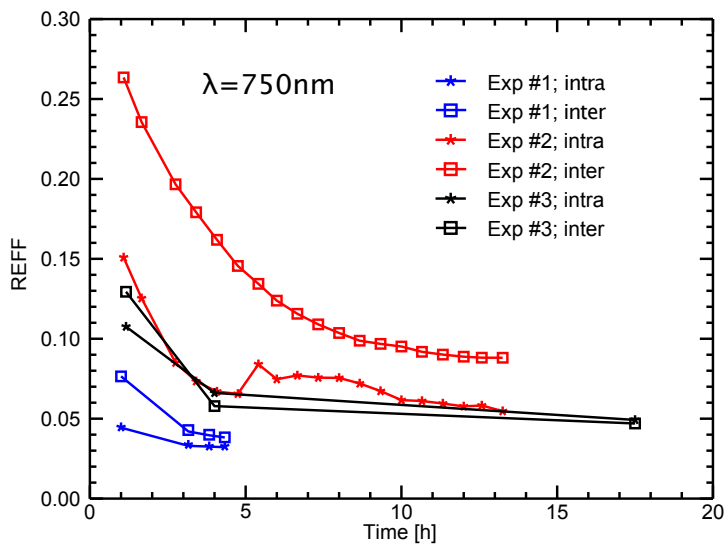

Figure 13: Evolution of the reflectance at $750 \mathrm{~nm}$ from all 3 experiments as a function of time. In all experiments the inter-mixture sample displays a higher reflectance than the intramixture. Experiment \#1 started already at very low reflectance, after $4 \mathrm{~h}$ the reflectance of the two mixtures becomes very similar. Experiment \#2 has the most complete dataset covering $13 \mathrm{~h}$. The inter mixture displays an exponential decrease in reflectance with time, but remains always brighter than the intra-mixture associated to its yellowish colour. The intra-mixture from this experiment shows a subtle increase of reflectance at $\sim 5 \mathrm{~h}$, this is a consequence of redistributed residue layer material where pristine bright material gets exposed. Experiment \#3 shows as well an exponential decrease of reflectance. The last point does not represent full $17 \mathrm{~h}$ of sublimation time, since the samples spent $\sim 2 \mathrm{~h}$ at ambient pressure in the goniometer and a storage freezer. 

a standard deviation as low as $0.57 \% / 100 \mathrm{~nm}$. This means that the relative spectral slope measured by hyperspectral imaging system is to high by a factor

There seems to be a problem in absolute calibration of the SCITEAS imaging system, observing at a phase angle of $13^{\circ}$. The dry tholin/charcoal powder (identical to the non-volatiles in the icy samples of this experiment) measured in experiment \#2 displays a relative spectral red slope of $\sim 21 \% / 100 \mathrm{~nm}$, while the same sample material measured by the PHIRE-2 goniometer has a relative slope of $\sim 6 \% / 100 \mathrm{~nm}$ at the corresponding phase angle. PHIRE-2 has been proven to have a very accurate absolute calibration compared to other instruments and photometric reference targets (see e.g. Pommerol et al., 2011). Despite the problematic absolute calibration, the individual measurements during the sublimation experiment are fairly reliable as the time sequence of the relative spectral slope from the (non-volatile) dry tholin/charcoal powder has 
of 3 but stable during all measurements. Further analysis of this issue will be necessary to fully understand these instrumental differences, and to accurately calibrate our data in absolute scale.

In all experiments the intra-mixtures have a higher relative spectral slope, i.e. they are redder, in units of $\%$ per $100 \mathrm{~nm}$, than the corresponding intermixtures at a specific time. All samples are becoming redder with time as the (bluer) water ice component disappears. As shown in Figs. 5 to 7 the intramixtures are always darker than the corresponding inter-mixtures. This might explain the differences in Fig. 11. Data of experiments \#1 and \#2 are fitted by linear functions. The rates of change of the mixtures from experiment \#2 seem very similar ( 0.8 vs. $1.0 \% / \mathrm{h})$, the ones from the other two experiments are more uncertain due to the low number of data points.

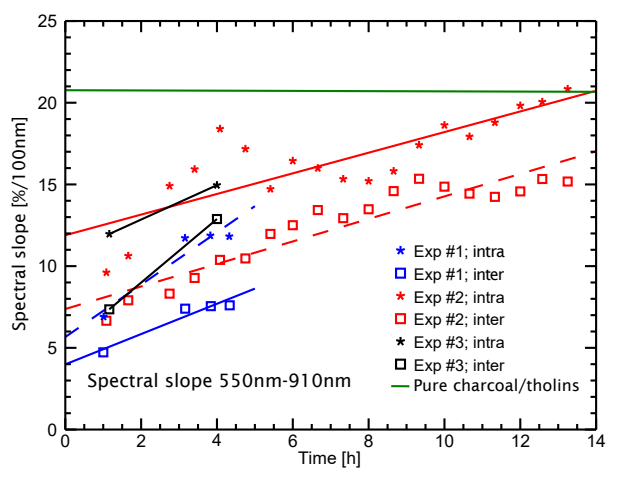

Figure 14: Relative spectral slope in units of \% per $100 \mathrm{~nm}$ in the $550-910 \mathrm{~nm}$ spectral range. The horizontal green line represents the spectral slope of a pure charcoal/tholin powder (90\%:10\%) which is the same composition as mixed with the icy samples in experiments \#2 and \#3. This powder was measured simultaneously during experiment \# 2, the 20 datapoints were averaged, having a standard deviation of $0.57 \% / 100 \mathrm{~nm}$. Experiments \#1 and \#2 are fitted with a linear function, experiment \#3 contains only 2 valid points, as the sublimation process was interrupted by $\sim 2 \mathrm{~h}$. In all experiments the intra-mixtures have a higher spectral slope than the corresponding inter-mixtures at a specific time.

To determine the amount of ice in the uppermost layer of the samples, the 
depth of the water absorption band is a good indicator. The spectrum of tholins and charcoal shows no absorption bands at the same wavelengths. The band depth is calculated as follows:

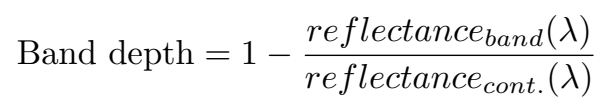

The reflectance at the band centre is divided by the reflectance of the continuum, connecting the adjacent local reflectance maxima (see illustration in Fig. 9) at the same wavelength. The measured band depths at 1.51 and $2.02 \mu \mathrm{m}$ are plotted as a function of time in Fig. 15. The depth as well as the rate of change seem very similar for the two bands. The behaviour of experiment \#2, which has a much higher initial albedo, appears different from the other experiments. It shows a clear difference between the band depths of intra- and inter-mixture at the beginning of the experiment, the inter-mixture showing deeper bands. After about $10 \mathrm{~h}$ the difference seems to vanish as the almost linear trends converge. The rates of changes of the darker samples from experiments \#1 and \#3 are by a factor of $2-3$ steeper compared to experiment \#2. Extrapolations would lead to a total disappearance of water ice bands after $5-6 \mathrm{~h}$ of sublimation time. This would be in general agreement with VIRTIS observations on $67 \mathrm{P}$ where the majority of surface areas does not show any evidence of water ice absorption features in the spectrum (Capaccioni et al., 2015), exceptions are bright spots (Barucci et al., 2016) and areas affected by diurnal recondensation cycles (De Sanctis et al., 2015).

After removing the samples from the vacuum chamber at the end of experiment \#3 a qualitative structural examination was performed to investigate the thickness of residue layer and solidification of sample material. At the stage of production all the samples had a powdery consistence with a low sintering and low cohesion tendency. It is well known that icy materials always strive to lower the total surface energy of the system and therefore reduce the total 

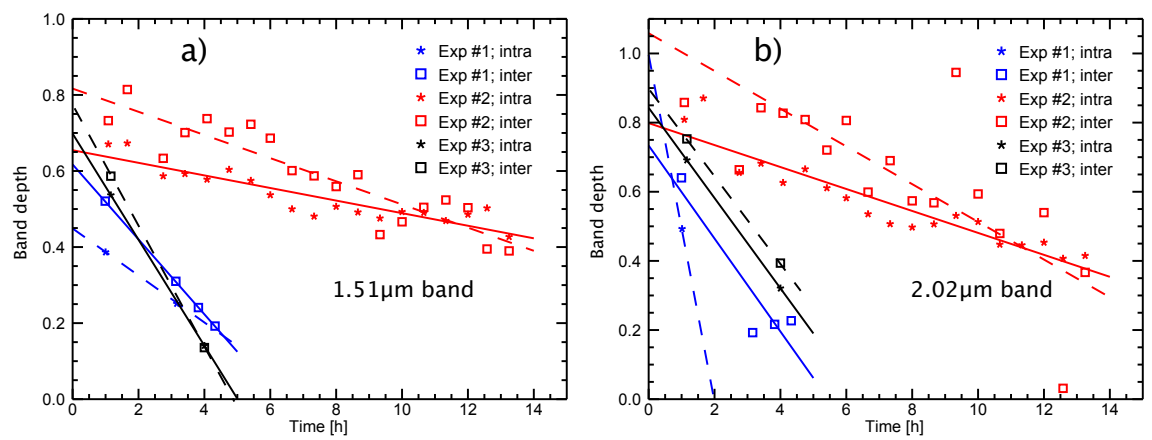

Figure 15: a) Band depths of the $1.51 \mu \mathrm{m}$ absorption band of water ice relative to a continuum at this wavelength, b) band depth at $2.02 \mu \mathrm{m}$ as a function of time. All samples show a decreasing trend of band depth, corresponding to a lower amount of water ice exposed at the surface. The rate of change from experiments \#1 and \#3 are similar while experiment \#2 has a lower rate due to its higher initial reflectance. The water ice band depth is mainly influenced by the carbon particles and not by the tholins which are nearly transparent in the NIR. In all experiments the inter-mixtures have a higher band depth than the intra-mixtures at a specific time, which is consistent with their slightly higher reflectance.

surface area by sintering of particle. Different transport mechanisms, such as vapour transport, are involved in this morphological change strongly dependent on temperature and pressure conditions (for more details see Jost et al., 2013 and references therein). Poch et al. (2016a,b) found that during sublimation experiments the inter-mixtures showed more consolidation throughout the entire bulk while intra-mixtures formed a hard crust below the residue mantle but the underlying material was still soft.

The examination of experiment \#3 was carried out by hand with a spatula cooled to liquid nitrogen temperature. The sample structure of pure ice was still quite loose without strong sintering effects, the upper part was slightly harder. The inter-mixture sample had built up a non-volatile mantle of $\sim 1 \mathrm{~mm}$ in the illuminated area, where some material was ejected, and $\sim 2 \mathrm{~mm}$ in the non-illuminated area. The texture of the mantle is powdery. Underneath the remaining ice was moderately hardened without a specific crust or vertical variation. The intra-mixture sample was covered by a residue layer of comparable 
thickness, but the mantle showed more internal cohesion and larger structures. The ice underneath formed a distinct, harder crust in the uppermost third of the bulk, but was still powdery below. The coarse-grained intra-mixture kept its granular surface texture from the beginning and formed no clear residue layer. It seemed that the porous mantle formed locally around big individual grains/agglomerates. Below the first monolayer of agglomerates $(\sim 2 \mathrm{~mm})$ a porous matrix of bright ice grains was found. Overall the sample was hardly sintered. These findings combined with the observation that on the coarse-grained intra-mixture only single particles were ejected or re-distributed across the surface lead to the interpretation that sublimation took place down to deeper layers or even the entire bulk. The rough structure allows gas flow from deeper layers to reach the surface trough a network of voids and channels. The smoother texture and more uniform grain size distribution of the normal intra-mixture performed and characterized with the SCITEAS and PHIRE-2 setups. These experiments under controlled laboratory conditions highlight the necessity of combining spectroscopy and bidirectional reflectance techniques in order to infer details about material composition as well as surface structure.

620

It was found that the two mixing types have slightly different spectra in the VIS range, the inter-mixtures generally being more yellowish have a more concave shaped spectrum and a higher reflectance. This is interpreted as a very efficient coating capability on nm-sized, orange-coloured tholin particles.

\section{Summary \& Conclusion}

In a series of laboratory investigations three sublimation experiments were In the NIR range the effects of mixing types are smaller as tholins are rather 
transparent in this spectral range. The biggest differences in terms of mixing types are observed on surface texture, gas driven activity and bulk consolidation. The intra-mixtures formed a filamentous, very porous matrix with high cohesion, resulting in higher gas impermeability and more violent ejection events. The residue of inter-mixture is more powdery, having lower cohesion producing smaller scaled ejections. This more compact residue mantle leads to enhanced downward flow of sublimation gas, recondensating in deeper layers. Thus the bulk gets more consolidated by recondensation.

We demonstrated the combined use of our two instrumental setups, SCITEAS and PHIRE-2, in all three experiments. The BRDF of unaltered samples was measured before inserting them in thermal vacuum during $6-14 \mathrm{~h}$ for sublimation under simulated space conditions. Afterwards the BRDF was measured again, showing reduced reflectance and lowered forward scattering behaviour, consistent with lower ice content and a more porous surface.

During experiment \#3 we have also demonstrated the integration of a solar simulator in our setup. The irradiation of the samples with visible light led to a different sublimation activity compared to the thermal IR flux reaching the 645 samples from the window of the chamber.

Spectroscopic analysis on all samples showed disappearing signatures of water ice in the NIR with time as the ice sublimed. This led as well to an increase of relative spectral slope in the VIS, i.e. the samples became redder, and the reflectance at $750 \mathrm{~nm}$ decreased.

All VIS-NIR imaging data from the nucleus of $67 \mathrm{P}$ acquired by Rosetta is affected by the composition and structure of the uppermost micrometres, as passive methods in this spectral range cannot penetrate deeper. A better understanding how the physical parameters, such as composition and structure of a refactory layer influences the observed spectra and phase curves is vital for 
remote sensing data interpretation.

Our experimental simulations have shown how the sublimation process leads

, B., Barbieri, C., Bockelee-Morvan, D., Lamy, P. L., De Sanctis C., Rodrigo, R., Erard, S., Koschny, D., Leyrat, C., Rickman, H., Drossart, P., Keller, H. U., A'Hearn, M. F., Arnold, G., Bertaux, J.-L., Bertini, I., Cerroni, P., Cremonese, G., Da Deppo, V., Davidsson, B. J. R., El-Maarry, Fonti, 

ice on the nucleus of comet $67 \mathrm{P} /$ Churyumov-Gerasimenko. ArXiv e-prints.

Bernard, J.-M., Quirico, E., Brissaud, O., Montagnac, G., Reynard, B., McMillan, P., Coll, P., Nguyen, M.-J., Raulin, F., Schmitt, B., Nov. 2006. Reflectance spectra and chemical structure of Titan's tholins: Application to the analysis of Cassini Huygens observations. Icarus 185, 301-307.

Brownlee, D., May 2014. The Stardust Mission: Analyzing Samples from the Edge of the Solar System. Annual Review of Earth and Planetary Sciences 42, 179-205.

Capaccioni, F., Coradini, A., Filacchione, G., Erard, S., Arnold, G., Drossart, P., De Sanctis, M. C., Bockelee-Morvan, D., Capria, M. T., Tosi, F., Leyrat, C., Schmitt, B., Quirico, E., Cerroni, P., Mennella, V., Raponi, A., Ciarniello, M., McCord, T., Moroz, L., Palomba, E., Ammannito, E., Barucci, M. A., Bellucci, G., Benkhoff, J., Bibring, J. P., Blanco, A., Blecka, M., Carlson, R., Carsenty, U., Colangeli, L., Combes, M., Combi, M., Crovisier, J., Encrenaz, T., Federico, C., Fink, U., Fonti, S., Ip, W. H., Irwin, P., Jaumann, R., Kuehrt, E., Langevin, Y., Magni, G., Mottola, S., Orofino, V., Palumbo, P., Piccioni, G., Schade, U., Taylor, F., Tiphene, D., Tozzi, G. P., Beck, P., Biver, N., Bonal, L., Combe, J.-P., Despan, D., Flamini, E., Fornasier, S., Frigeri, A., Grassi, D., Gudipati, M., Longobardo, A., Markus, K., Merlin, F., Orosei, R., Rinaldi, G., Stephan, K., Cartacci, M., Cicchetti, A., Giuppi, S., Hello, Y., Henry, F., Jacquinod, S., Noschese, R., Peter, G., Politi, R., Reess, J. M., Semery, A., Jan. 2015. The organic-rich surface of comet 67P/ChuryumovGerasimenko as seen by VIRTIS/Rosetta. Science 347 (1), aaa0628.

Carrasco, N., Schmitz-Afonso, I., Bonnet, J.-Y., Quirico, E., Thissen, R., Dutuit, O., Bagag, A., Laprévote, O., Buch, A., Giulani, A., Adandé, G., Ouni, F., Hadamcik, E., Szopa, C., Cernogora, G., Sep. 2009. Chemical Characterization of Titan's Tholins: Solubility, Morphology and Molecular Structure Revisited. Journal of Physical Chemistry A 113, 11195-11203. 
Clark, R. N., Carlson, R., Grundy, W., Noll, K., 2013. The Science of Solar Ices in the Solar System, pp. 3-46.

de Bergh, C., Schmitt, B., Moroz, L. V., Quirico, E., Cruikshank, D. P., 2008. Laboratory Data on Ices, Refractory Carbonaceous Materials, and Minerals Relevant to Transneptunian Objects and Centaurs. pp. 483-506.

De Sanctis, M. C., Capaccioni, F., Ciarniello, M., Filacchione, G., Formisano, M., Mottola, S., Raponi, A., Tosi, F., Bockelée-Morvan, D., Erard, S., Leyrat, C., Schmitt, B., Ammannito, E., Arnold, G., Barucci, M. A., Combi, M., Capria, M. T., Cerroni, P., Ip, W.-H., Kuehrt, E., McCord, T. B., Palomba, E., Beck, P., Quirico, E., VIRTIS Team, Piccioni, G., Bellucci, G., Fulchignoni, M., Jaumann, R., Stephan, K., Longobardo, A., Mennella, V., Migliorini, A., Benkhoff, J., Bibring, J. P., Blanco, A., Blecka, M., Carlson, R., Carsenty, U., Colangeli, L., Combes, M., Crovisier, J., Drossart, P., Encrenaz, T., Federico, C., Fink, U., Fonti, S., Irwin, P., Langevin, Y., Magni, G., Moroz, L., Orofino, V., Schade, U., Taylor, F., Tiphene, D., Tozzi, G. P., Biver, N., Bonal, L., Combe, J.-P., Despan, D., Flamini, E., Fornasier, S., Frigeri, A., Grassi, D., Gudipati, M. S., Mancarella, F., Markus, K., Merlin, F., Orosei, R., Rinaldi, G., Cartacci, M., Cicchetti, A., Giuppi, S., Hello, Y., Henry, F., Jacquinod, S., Rees, J. M., Noschese, R., Politi, R., Peter, G., Sep. 2015 . The diurnal cycle of water ice on comet $67 \mathrm{P} /$ Churyumov-Gerasimenko. Nature 525, 500-503.

Dobrovolsky, O. V., Kaimakov, E. A., 1977. Surface phenomena in simulated cometary nuclei. In: Delsemme, A. H. (Ed.), IAU Colloq. 39: Comets, Asteroids, Meteorites: Interrelations, Evolution and Origins. pp. 37-45.

Feller, C., Fornasier, S., Hasselmann, P. H., Barucci, A., Preusker, F., Scholten, F., Jorda, L., Pommerol, A., Sierks, H., Agarwal, J., A'Hearn, M., Bertaux, J.-L., Bertini, I., Boudreault, S., Cremonese, G., Da Deppo, V., Davidsson, B. J. R., Debei, S., De Cecco, M., Deller, J., Fulle, M., Giquel, A., Groussin, 
O., Gutierrez, P. J., Güttler, C., Hofmann, M., Hviid, S. F., Keller, H., Ip, W.-H., Knollenberg, J., Kovacs, G., Kramm, J.-R., Kührt, E., Küppers, M., Lara, M. L., Lazzarin, M., Leyrat, C., Lopez Moreno, J. J., Marzari, F., Masoumzadeh, N., Mottola, S., Naletto, G., Oklay, N., Shi, X., Tubiana, C., Vincent, J.-B., 2016. Decimetre-scaled spectrophotometric properties of the nucleus of comet $67 \mathrm{P} /$ Churyumov-Gerasimenko from OSIRIS observations. Monthly Notices of the Royal Astronomical Society 462 (Suppl 1), S287-S303.

Fornasier, S., Hasselmann, P. H., Barucci, M. A., Feller, C., Besse, S., Leyrat, C., Lara, L., Gutierrez, P. J., Oklay, N., Tubiana, C., Scholten, F., Sierks, H., Barbieri, C., Lamy, P. L., Rodrigo, R., Koschny, D., Rickman, H., Keller, H. U., Agarwal, J., A’Hearn, M. F., Bertaux, J.-L., Bertini, I., Cremonese, G., Da Deppo, V., Davidsson, B., Debei, S., De Cecco, M., Fulle, M., Groussin, O., Güttler, C., Hviid, S. F., Ip, W., Jorda, L., Knollenberg, J., Kovacs, G., Kramm, R., Kührt, E., Küppers, M., La Forgia, F., Lazzarin, M., Lopez Moreno, J. J., Marzari, F., Matz, K.-D., Michalik, H., Moreno, F., Mottola, S., Naletto, G., Pajola, M., Pommerol, A., Preusker, F., Shi, X., Snodgrass, C., Thomas, N., Vincent, J.-B., Nov. 2015. Spectrophotometric properties of the nucleus of comet $67 \mathrm{P} /$ Churyumov-Gerasimenko from the OSIRIS instrument onboard the ROSETTA spacecraft. Astronomy \& Astrophysics 583, A30.

Gautier, T., Carrasco, N., Schmitz-Afonso, I., Touboul, D., Szopa, C., Buch, A., Pernot, P., Oct. 2014. Nitrogen incorporation in Titan's tholins inferred by high resolution orbitrap mass spectrometry and gas chromatography-mass spectrometry. Earth and Planetary Science Letters 404, 33-42.

Gautier, T., Schmitz-Afonso, I., Touboul, D., Szopa, C., Buch, A., Carrasco, N., Sep. 2016. Development of HPLC-Orbitrap method for identification of N-bearing molecules in complex organic material relevant to planetary environments. Icarus 275, 259-266.

Gruen, E., Bar-Nun, A., Benkhoff, J., Bischoff, A., Dueren, H., Hellmann, H., Hesselbarth, P., Hsiung, P., Keller, H. U., Klinger, J., 1991. Laboratory 
simulation of cometary processes - Results from first KOSI experiments. In: Newburn, Jr., R. L., Neugebauer, M., Rahe, J. (Eds.), IAU Colloq. 116: Comets in the post-Halley era. Vol. 167 of Astrophysics and Space Science Library. pp. 277-297.

Gruen, E., Gebhard, J., Bar-Nun, A., Benkhoff, J., Dueren, H., Eich, G., Hische, R., Huebner, W. F., Keller, H. U., Klees, G., Aug. 1993. Development of a dust mantle on the surface of an insolated ice-dust mixture - Results from the KOSI-9 experiment. Journal of Geophysical Research: Planets 98, 15.

780 Jost, B., Gundlach, B., Pommerol, A., Oesert, J., Gorb, S. N., Blum, J., Thomas, N., 2013. Micrometer-sized ice particles for planetary-science experiments II. Bidirectional reflectance. Icarus 225 (1), $352-366$.

Jost, B., Pommerol, A., Poch, O., Fornasier, S., Carrasco, N., Szopa, C., Thomas, N., submitted. Bidirectional re ectance of laboratory cometary analogues to interpret the spectrophotometric properties of the nucleus of comet 67P/Churyumov-Gerasimenko. Planetary and Space Science.

Jost, B., Pommerol, A., Poch, O., Gundlach, B., Leboeuf, M., Dadras, M., Blum, J., Thomas, N., Jan. 2016. Experimental characterization of the opposition surge in fine-grained water-ice and high albedo ice analogs. Icarus 264, 109-131.

Mahjoub, A., Carrasco, N., Dahoo, P.-R., Gautier, T., Szopa, C., Cernogora, G., Nov. 2012. Influence of methane concentration on the optical indices of Titan's aerosols analogues. Icarus 221, 670-677.

Mastrapa, R. M. E., Grundy, W. M., Gudipati, M. S., 2013. The Science of Solar System Ices. Springer New York, New York, NY, Ch. Amorphous and Crystalline $\mathrm{H}_{2} \mathrm{O}-\mathrm{Ice}$, pp. 371-408.

McDonald, G. D., Whited, L. J., DeRuiter, C., Khare, B. N., Patnaik, A., Sagan, C., 1996. Production and chemical analysis of cometary ice tholins. Icarus 122 (1), 107 - 117. 
Oehler, A., Neukum, G., Feb. 1991. Visible and near IR albedo measurements of ice/dust mixtures. Geophysical Research Letters 18, 253-256.

Pernot, P., Carrasco, N., Thissen, R., Schmitz-Afonso, I., Jan. 2010. Tholinomics - chemical analysis of nitrogen-rich polymers. Analytical Chemistry $82,1371-1380$.

Poch, O., Pommerol, A., Jost, B., Carrasco, N., Szopa, C., Thomas, N., Mar. 2016a. Sublimation of ice-tholins mixtures: A morphological and spectrophotometric study. Icarus 266, 288-305.

Poch, O., Pommerol, A., Jost, B., Carrasco, N., Szopa, C., Thomas, N., Mar. 2016b. Sublimation of water ice mixed with silicates and tholins: Evolution of surface texture and reflectance spectra, with implications for comets. Icarus $267,154-173$.

Pommerol, A., Jost, B., Poch, O., El-Maarry, M. R., Vuitel, B., Thomas, N., May 2015a. The SCITEAS experiment: Optical characterizations of sublimating icy planetary analogues. Planetary and Space Science 109, 106-122.

815 Pommerol, A., Thomas, N., Affolter, M., Portyankina, G., Jost, B., Seiferlin, K., Aye, K.-M., Oct. 2011. Photometry and bulk physical properties of Solar System surfaces icy analogs: The Planetary Ice Laboratory at University of Bern. Planetary and Space Science 59, 1601-1612.

Quirico, E., Montagnac, G., Lees, V., McMillan, P. F., Szopa, C., Cernogora, G., Rouzaud, J.-N., Simon, P., Bernard, J.-M., Coll, P., Fray, N., Minard, R. D., Raulin, F., Reynard, B., Schmitt, B., Nov. 2008. New experimental constraints on the composition and structure of tholins. Icarus 198, 218-231.

Sagan, C., Khare, B. N., Jan. 1979. Tholins - Organic chemistry of interstellar grains and gas. Nature 277, 102-107.

825 Sandford, S. A., Aléon, J., Alexander, C. M. O. ., Araki, T., Bajt, S., Baratta, G. A., Borg, J., Bradley, J. P., Brownlee, D. E., Brucato, J. R., Burchell, 
M. J., Busemann, H., Butterworth, A., Clemett, S. J., Cody, G., Colangeli, L., Cooper, G., D'Hendecourt, L., Djouadi, Z., Dworkin, J. P., Ferrini, G., Fleckenstein, H., Flynn, G. J., Franchi, I. A., Fries, M., Gilles, M. K., Glavin, D. P., Gounelle, M., Grossemy, F., Jacobsen, C., Keller, L. P., Kilcoyne, A. L. D., Leitner, J., Matrajt, G., Meibom, A., Mennella, V., Mostefaoui, S., Nittler, L. R., Palumbo, M. E., Papanastassiou, D. A., Robert, F., Rotundi, A., Snead, C. J., Spencer, M. K., Stadermann, F. J., Steele, A., Stephan, T., Tsou, P., Tyliszczak, T., Westphal, A. J., Wirick, S., Wopenka, B., Yabuta, H., Zare, R. N., Zolensky, M. E., Dec. 2006. Organics Captured from Comet 81P/Wild 2 by the Stardust Spacecraft. Science 314, 1720.

Saunders, R. S., Fanale, F. P., Parker, T. J., Stephens, J. B., Sutton, S., Apr. 1986. Properties of filamentary sublimation residues from dispersions of clay in ice. "Icarus" 66, 94-104.

840 Sciamma-O'Brien, E., Carrasco, N., Szopa, C., Buch, A., Cernogora, G., 2010. Titan's atmosphere: an optimal gas mixture for aerosol production? Icarus 209 (2), 704-714.

Seiferlin, K., Spohn, T., Benkhoff, J., Jul. 1995. Cometary ice texture and the thermal evolution of comets. Advances in Space Research 15, 35-38.

${ }_{845}$ Spohn, T., Knollenberg, J., Ball, A. J., Banaszkiewicz, M., Benkhoff, J., Grott, M., Grygorczuk, J., Hüttig, C., Hagermann, A., Kargl, G., Kaufmann, E., Kömle, N., Kührt, E., Kossacki, K. J., Marczewski, W., Pelivan, I., Schrödter, R., Seiferlin, K., Jul. 2015. Thermal and mechanical properties of the nearsurface layers of comet 67P/Churyumov-Gerasimenko. Science 349 (2).

Storrs, A. D., Fanale, F. P., Saunders, R. S., Stephens, J. B., Dec. 1988. The formation of filamentary sublimate residues (FSR) from mineral grains. "Icarus" $76,493-512$.

Szopa, C., Cernogora, G., Boufendi, L., Correia, J. J., Coll, P., Apr. 2006. PAMPRE: A dusty plasma experiment for Titan's tholins production and study. Planetary and Space Science 54, 394-404. 
Wooden, D. H., 2008. Cometary Refractory Grains: Interstellar and Nebular Sources. Springer New York, New York, NY, pp. 75-108.

Yoldi, Z., Pommerol, A., Jost, B., Poch, O., Gouman, J., Thomas, N., Aug. 2015. VIS-NIR reflectance of water ice/regolith analogue mixtures and implications for the detectability of ice mixed within planetary regoliths. Geophysical Research Letters 42, 6205-6212. 